\section{International Scientific Journal Theoretical \& Applied Science}

p-ISSN: 2308-4944 (print) e-ISSN: 2409-0085 (online)

Year: $2016 \quad$ Issue: 4 Volume: 36

Published: $30.04 .2016 \quad$ http://T-Science.org

SECTION 5. Innovative technologies in science.
S. Sheraliyev

Senior Researcher, National University of Uzbekistan, Tashkent, Uzbekistan sadulla72@mail.ru

O. Tigay

Associate Professor, Ph.D., Tashkent University of Information Technologies, Tashkent, Uzbekistan

Sh. Abdurakhmanova

Teacher, Tashkent State Pedagogical University named after Nizami, Tashkent, Uzbekistan

S. Alibekov

Senior Lecturer, Ph.D. Tashkent State Pedagogical University named after Nizami, Tashkent, Uzbekistan

\title{
INTEGRATED TECHNIQUE FOR SOLVING PROBLEMS IN PHYSICS USING MATHCAD TOOLKIT AND CROCODILE TECHNOLOGY 3D
}

Abstract: This article discusses an integrated approach to solving problems in physics using modern information technology, which allows students to study further specified conditions, to master various techniques solutions, such as physical and mathematical modeling on a computer, substantiate the choice of the most appropriate combination of methods.

Key words: simulation of physical processes, mathematical modeling, simulation tasks, virtual experiment, integrated problem-solving methods.

Language: Russian

Citation: Sheraliyev S, Tigay O, Abdurakhmanova S, Alibekov S (2016) INTEGRATED TECHNIQUE FOR SOLVING PROBLEMS IN PHYSICS USING MATHCAD TOOLKIT AND CROCODILE TECHNOLOGY 3D. ISJ Theoretical \& Applied Science, 04 (36): 101-105.

Soi: http://s-o-i.org/1.1/TAS-04-36-14 Doi: crossef http://dx.doi.org/10.15863/TAS.2016.04.36.14

\section{ИНТЕГРИРОВАННАЯ МЕТОДИКА РЕШЕНИЯ ЗАДАЧ ПО ФИЗИКЕ С ПРИМЕНЕНИЕМ ПРОГРАММ МАТНСАD И CROCODILE TECHNOLOGY 3D}

Аннотация: В статье рассматривается интегрированный подход при решении задач по физике с использованием современных информационных технологий, который позволяет учащимся более детально изучить заданные условия, овладеть различными методами решения, такими. Как физическое и математическое моделирование на компьютере, обосновать выбор наиболее подходящей комбинации методов.

Ключевые слова: симулятор физических прочессов, математическое моделирование, моделирование задачи, виртуальный эксперимент, интегрированные методы решения задач.

Введение. На практических занятиях по физике вербальное знание переходит в умение и навык, приобретает четкость, определенность. Решение задач превращается в главное средство обучения, происходит дифференцирование исходного знания, оно наполняется частными деталями [1-3]. Этот этап должен осуществляться при минимальной помощи со стороны преподавателя или даже при полном еe отсутствии.

Интегрированный подход в решении задач по физике с использованием MathCAD и Crocodile Technology 3D, дает возможность студентам и учащимая глубже понять физический смысл задачи, освоить принципы анализа условий, самостоятельно закрепить и усовершенствовать методы решения задач и ознакомиться с новыми методами решения, такие как проектирование и моделирование задач на компьютере, а также проверить правдоподобность результатов.

MathCAD является математическим редактором, позволяющим проводить разнообразные научные и инженерные расчеты, начиная от элементарной арифметики и заканчивая сложными реализациями численных методов. Благодаря простоте применения, наглядности математических действий, 
обширной библиотеке встроенных функций и численных методов, возможности символьных вычислений, а также превосходному аппарату представления результатов (графики самых разных типов, мощных средств подготовки печатных документов и Web-страниц), MathCAD стал наиболее популярным математическим приложением. В отличие от большинства других современных математических приложений, он построен в соответствии с принципом WYSIWYG ("What You See Is What You Get" "что Вы видите, то и получите"). Поэтому он очень прост в использовании, в частности, из-за отсутствия необходимости написания программы для расчетов[4-9].

Crocodile Technology 3D объединяет в себе электронный проект, программирование PIC, механизмы 3D и моделирование $3 \mathrm{D}$ PCB. Crocodile Technology 3D - 3D симулятор электронных цепей, с помощью которого можно разработать принципиальную электрическую схему устройства, монтажную плату под него и т.д.. Crocodile Technology 3D используется в учебном процессе как платформа виртуальных экспериментов, позволяющая студентам проводить эксперименты и изучать различные темы в процессе обучения, а также рекомендуется в качестве приложения к программированию, электронике, механике и другим подобным курсам. [10]

Основные вопросы курса. При самостоятельном решении задач по физике студент или учащихся последовательно на первоначальном этапе решает задачу с помощью методов и приемов, основанных на знании теоретического материала, соответствующих правилах и законах, то есть традиционным способом.

\section{Рассмотрим пример.}

Определить силу тока в сопротивлении $R_{3} u$ напряжение на концах этого сопротивления. $E_{1}=1 B, E_{2}=5 B, R_{1}=1 O M, R_{2}=2 O M, R_{3}=9 O_{M}$. (Рис.1)

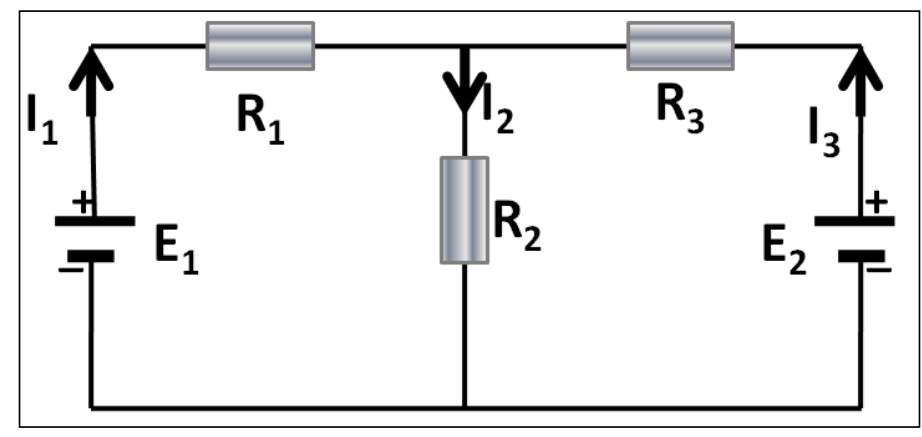

Рисунок 1 - Схема.

1 Этап. Для решения задачи используем правила Кирхгофа.

$\left\{\begin{array}{c}I_{1}-I_{2}+I_{3}=0 \\ I_{1} R_{1}+I_{2} R_{2}=E_{1} \\ I_{1} R_{1}-I_{3} R_{2}=E_{1}-E_{2}\end{array} \Rightarrow\left\{\begin{array}{c}I_{1}-I_{2}+I_{3}=0 \\ I_{1}+2 I_{2}+0=1 \\ I_{1}+0-9 I_{3}=-4\end{array}\right.\right.$

После решения системы получаем:

$$
\mathrm{I}_{1}=34 \mathrm{~mA}, \quad \mathrm{I}_{2}=482 \mathrm{~mA}, \quad \mathrm{I}_{3}=448 \mathrm{~mA}
$$

напряжение $\mathrm{U}_{3}$ найдем по закону Ома для участка цепи:

$$
\mathrm{U}_{3}=\mathrm{I}_{3} \mathrm{R}_{3}=0,448 \mathrm{~A} \cdot 9 \text { OM }=4,03 \mathrm{~B} .
$$

2 Этап. Математическое моделирование в MathCAD (Рис.2). На этом этапе, уже имея предварительные результаты, студент или учащихся математически моделирует задачу в MathCAD, используя метод Крамера, вводит расчетные формулы и исходные данные с помощью встроенного редактора формул, запускает исполнение программы, которая реализует математические расчеты и выдает их в виде численных или графических значений. На данном этапе можно подтвердить или опровергнуть ранее полученные результаты. 


\begin{tabular}{|c|c|c|c|c|c|c|}
\hline Impact Factor: & $\begin{array}{l}\text { ISRA (India) } \\
\text { ISI (Dubai, UAF } \\
\text { GIF (Australia) } \\
\text { JIF }\end{array}$ & $\begin{array}{l}=1.344 \\
=0.829 \\
=0.564 \\
=1.500\end{array}$ & $\begin{array}{l}\text { SIS (USA) } \\
\text { PИНЦ (Russia) } \\
\text { ESJI (KZ) } \\
\text { SJIF (Morocco) }\end{array}$ & $\begin{array}{l}=0.912 \\
=0.234 \\
=1.042 \\
=\mathbf{2 . 0 3 1}\end{array}$ & $\begin{array}{l}\text { ICV (Poland) } \\
\text { PIF (India) } \\
\text { IBI (India) }\end{array}$ & $\begin{array}{l}=6.630 \\
=1.940 \\
=4.260\end{array}$ \\
\hline
\end{tabular}

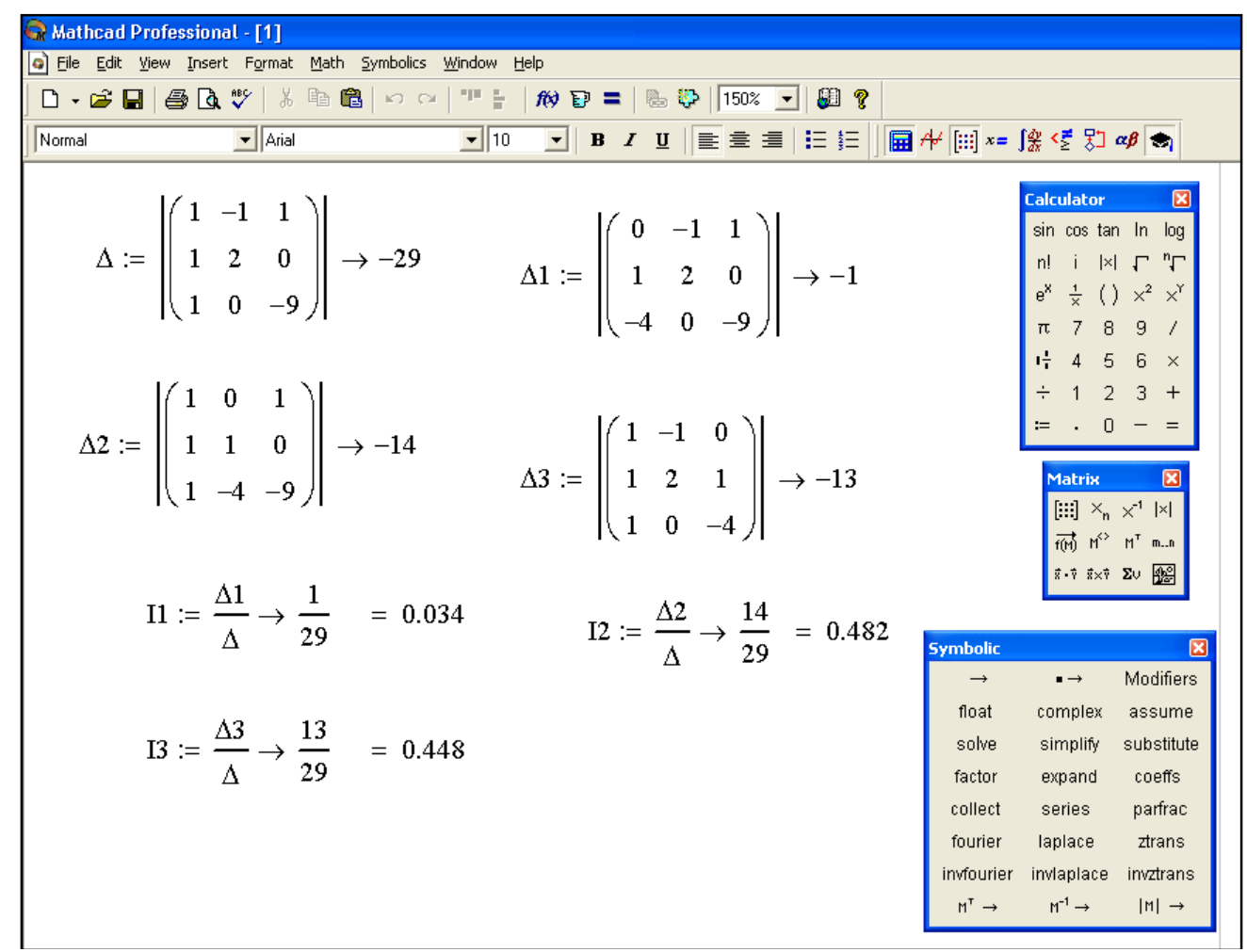

Рисунок 2 - Математическое моделирование в MathCAD.

3 Этап. Моделирование в Crocodile Technology 3D (Рис.2). Этот этап начинается c разработки и моделирования задачи при помощи Crocodile Technology 3D симулятора. Схема собирается с использованием необходимого набора конструктивных элементов в соответствии с правилами сборки электрических цепей и данными решаемой задачи. Уже в процессе сборки все измерительные и регистрирующие приборы показывают численные значения измеряемых величин, которые при правильном составлении схемы должны совпадать с теоретическими расчетами, полученными ранее другими методами.

Parts Library
Analogue
Digectangular PCB space
Digital 4000 Series
Digital 7400 Series
Standard Bloctrocks PCB space
Flowcharts
Mechanisms

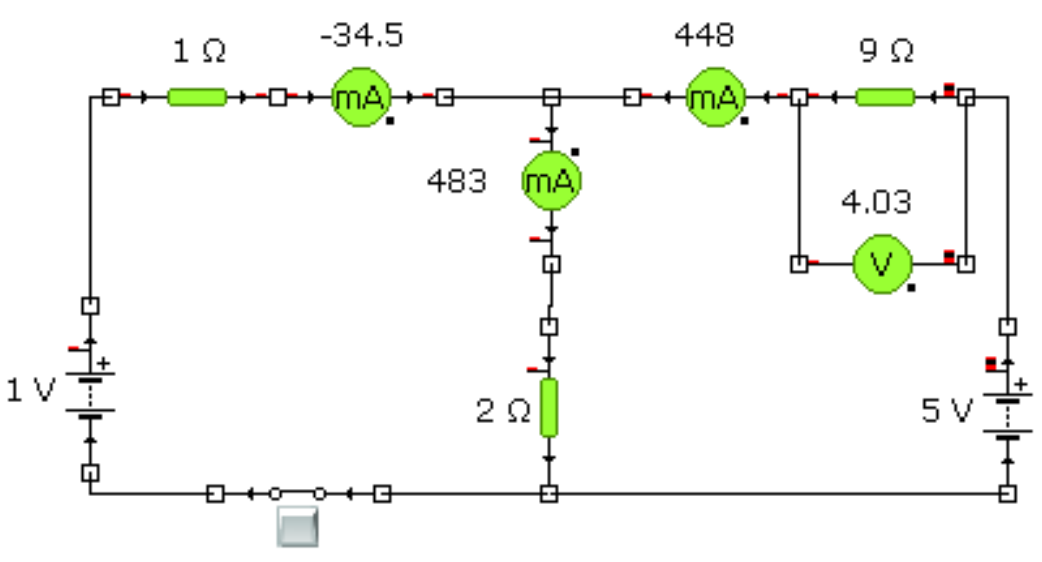

Рисунок 3 - Симулятор.

ISPC Global Science, 


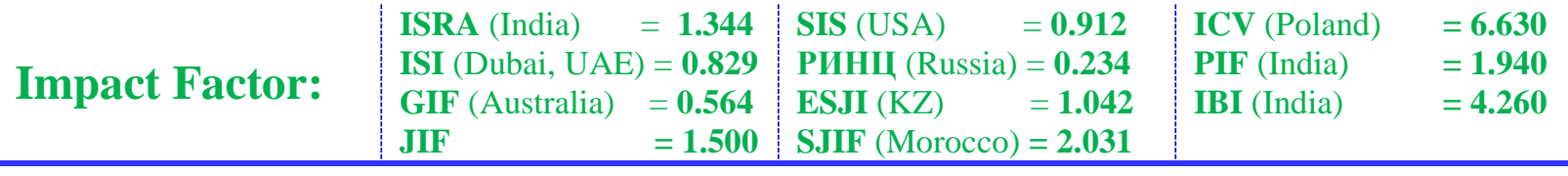

Завершающим этапом такого моделирования является компилирование программой и выдача 3D модели собранной схемы на монтажной плате, с наиболее рациональным размещением и соединением деталей, что позволяет студенту наглядно изучить рассчитанную схему (Рис.4).
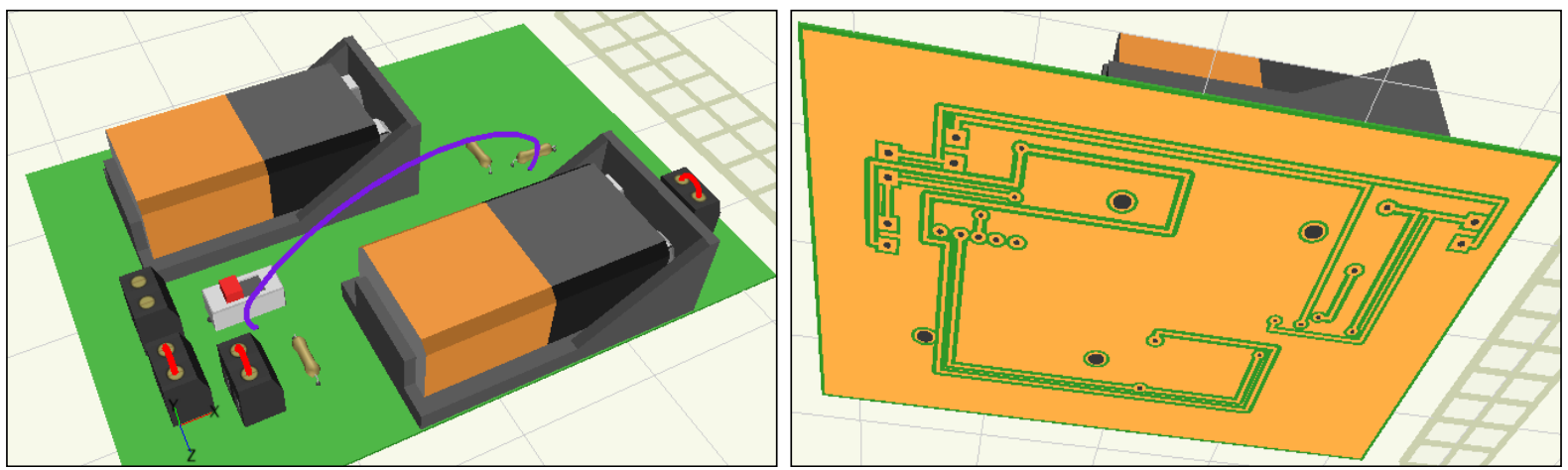

Рисунок 4 - 3D модель собранной схемы.

Заключение. Таким образом, при решении задач по физике по данной методике, студент осваивает довольно сложный, но необходимый комплекс действий:

- умение осознать смысл условия задачи, выявить скрытые данные, определить характер описываемого явления, главные и второстепенные факторы, понять и конкретизировать содержание главного вопроса, построить модель;

- гибкое владение различными методами решения, в том числе и навыками физического и математического моделирования, умение сделать и обосновать выбор наиболее подходящей комбинации методов;
- умение работать с учебными прикладными программами, электронными симуляторами и конструкторами;

- безошибочное применение и обоснованное видоизменение методов решения с учетом конкретных условий и ограничений, как задачи, так и самих методов;

- вносить изменения в исходные данные задач, умение анализировать результаты и оценивать их физический смысл.

Авторы выражают блогадарность за советы и критические замечаниеи научному консультанту член коор. МАНПО К.А. Турсунметову.

\section{References:}

1. Tursunmetov KA, Sheraliev SS, Mavlyanov K (2011) Uzbekskiy zhurnal PROBLEMY INFORMATIKI i ENERGETIKI. Tashkent, 2011. №5. -88 p. Nauchno - tekhnicheskie aspekty primeneniya animatsionnykh programm v nauchnykh i uchebnykh razrabotok (obzor).

2. Umarova LK (2004) K voprosu ob ispol'zovanii komp'yuternogo modelirovaniya pri reshenii fizicheskikh zadach.// Nauchnye trudy MPGU. Ser. Estestvennye nauki. Sbornik stat'ey. Moscow: Prometey, 2004. - pp. 140-143.

3. Izvozchikov VA, Slutskiy AM (1999) reshenie zadach po fizike na komp'yutere: kniga dlya uchitelya. - Moscow: Prosveshchenie, 1999. 254 p.

4. Ravshanov N, Shertaev M, Toshtemirova N (2015) Mathematical Model for the Study and
Forecast of the Concentration of Harmful Substances in the Atmosphere // American Journal of Modeling and Optimization. - 2015. Vol. 3. - № 2. - pp. 35-39.

5. Ravshanov N, Sharipov DK, Akhmedov D (2015) Modeling pollution process environment, taking into account the terrain climatic factors // Information technology modeling and management - Voronezh, 2015. №3. - pp. 222-235.

6. Sharipov DK (2013) Development of mathematical software aerosol transport and diffusion of the atmospheric emissions // European Applied Sciences. - 2013. -Vol. 1. № 1. - pp. 233-240.

7. Sharipov D (2016) A Mathematical Model and Computational Experiment for the Study and Forecast of the Concentration of Harmful 


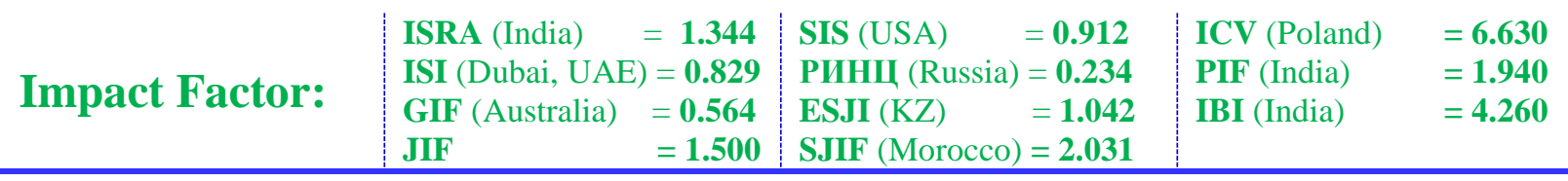

Substances in the Atmosphere // American Journal of Computation, Communication and Control. - 2016. - № 2 (6). - pp. 48-54.

8. Ravshanov N, Sharipov DK, Narzullaeva N (2016) advanced mathematical model of the processes of transfer and diffusion of pollutants in the surface atmospheric boundary layer // Scientific Review. Technical science. - Penza: Natural Science Academy, 2016. - № 4. - pp. 49-59.
9. Ravshanov N, Sharipov D, Muradov F (2016) Computational Experiment For Forecasting And Monitoring The Environmental Condition Of Industrial Regions. ISJ Theoretical \& Applied Science, 03 (35): 132-139.

10. Ibragimova DK, Shalkharova ZS, Sadykova AD, Zhunisova MB, Sadykova KZ, Shalkharova ZN (2016) The Mortality Rate Depending On Gender, Ethnicity And Risk Factors In Turkestan Region. ISJ Theoretical \& Applied Science, 03 (35): 176-181. 